\title{
Therapeutic effects of the novel subtype-selective histone deacetylase inhibitor chidamide on myeloma-associated bone disease
}

\begin{abstract}
Jingsong He, ${ }^{1 *}$ Qingxiao Chen,,$^{1 *}$ Huiyao Gu, ${ }^{1}$ Jing Chen, ${ }^{1}$ Enfan Zhang, ${ }^{1}$ Xing Guo, ${ }^{1}$ Xi Huang, ${ }^{1}$ Haimeng Yan, ${ }^{1}$ DongHua He, ${ }^{1}$ Yang Yang, ${ }^{1}$ Yi Zhao, ${ }^{1}$ Gang Wang, ${ }^{1,2}$ Huang $\mathrm{He}^{1}{ }^{1}$ Qing $\mathrm{Yi}^{3}$ and Zhen Cai ${ }^{1}$
\end{abstract}

${ }^{1}$ Bone Marrow Transplantation Center, Department of Hematology, The First Affiliated Hospital, School of Medicine, Zhejiang University, Hangzhou, China; '2Quzhou People's Hospital, Zhejiang Province, China and ${ }^{3}$ Department of Cancer Biology, Lerner Research Institute, Cleveland Clinic, OH, USA

\section{ABSTRACT}

$\mathrm{H}$ istone deacetylases are promising therapeutic targets in hematological malignancies. In the work herein, we investigated the effect of chidamide, a new subtype-selective histone deacetylase inhibitor that was independently produced in China, on multiple myeloma and its associated bone diseases using different models. The cytotoxicity of chidamide toward myeloma is due to its induction of cell apoptosis and cell cycle arrest by increasing the levels of caspase family proteins p21 and p27, among others. Furthermore, chidamide exhibited significant cytotoxicity against myeloma cells co-cultured with bone mesenchymal stromal cells and chidamide-pretreated osteoclasts. Importantly, chidamide suppressed osteoclast differentiation and resorption in vitro by dephosphorylating p-ERK, p-p38, p-AKT and p-JNK and inhibiting the expression of Cathepsin K, NFATc1 and c-fos. Finally, chidamide not only prevented tumor-associated bone loss in a disseminated murine model by partially decreasing the tumor burden but also prevented rapid receptor activator of nuclear factor $\kappa-\beta$ ligand (RANKL)-induced bone loss in a non-tumor-bearing mouse model. Based on our results, chidamide exerted dual anti-myeloma and bone-protective effects in vitro and in vivo. These findings strongly support the potential clinical use of this drug as a treatment for multiple myeloma in the near future.

\section{Introduction}

Multiple myeloma (MM) is an incurable plasma malignancy characterized by the accumulation of monoclonal plasma cells in the bone marrow (BM), the secretion of high levels of monoclonal immunoglobulins and osteolytic bone lesions. ${ }^{1}$ Myeloma cells interact with different cell types in the BM microenvironment, such as osteoclasts (OCs) and mesenchymal stromal cells, which supports their growth, drug resistance and the development of bone disease through cell-cell adhesion and the release of growth factors, such as interleukin-6 (IL-6) and vascular endothelial growth factor (VEGF). ${ }^{1}$ Osteolytic bone lesions, which result from increased bone resorption by OCs and reduced osteoblastic bone formation, are the most common complication of $\mathrm{MM}$ and often decrease the quality of life and survival time of patients with MM.,3 New active drugs, including proteasome inhibitors and immunomodulatory agents, have improved the outcomes of patients with $\mathrm{MM}$ and alleviated bone damage. ${ }^{1}$ However, disease progression is still inevitable. Therefore, the identification of new targets and development of new drugs that focus on $\mathrm{MM}$ cells and their BM microenvironment are crucial to the development of more effective treatments.

Chidamide is a novel oral histone deacetylase inhibitor (HDACi) designed to inhibit the activity of HDAC1, 2, 3 and 10 which is produced independently in China and is now undergoing phase I clinical trials in America and Japan.,
Haematologica 2018

Volume 103(8):1369-1379

\section{Correspondence:}

caiz@zju.edu.cn

Received: November 8, 2017

Accepted: April 27, 2018.

Pre-published: May 17, 2018.

doi:10.3324/haematol.2017.181172

Check the online version for the most updated information on this article, online supplements, and information on authorship \& disclosures: www.haematologica.org/content/103/8/1369

\section{(C)2018 Ferrata Storti Foundation}

Material published in Haematologica is covered by copyright. All rights are reserved to the Ferrata Storti Foundation. Use of published material is allowed under the following terms and conditions:

https://creativecommons.org/licenses/by-nc/4.0/legalcode. Copies of published material are allowed for personal or internal use. Sharing published material for non-commercial purposes is subject to the following conditions:

https://creativecommons.org/licenses/by-nc/4.0/legalcode, sect. 3. Reproducing and sharing published material for commercial purposes is not allowed without permission in writing from the publisher. 
Chidamide has been shown to induce cell apoptosis and cell cycle arrest in a variety of cancers, such as lung cancer, pancreatic cancer, leukemia and lymphoma. ${ }^{47}$ Moreover, HDAC3 was recently shown to be overexpressed in MM and was a key factor that propelled myeloma cell proliferation. ${ }^{8,9}$ Additionally, HDAC1 and HDAC2 are important positive regulators of osteoclastogenesis. ${ }^{10,11}$ Based on these data, we hypothesize that chidamide may exert dual anti-myeloma and bone-protective effects.

In the work herein, we investigated the dual antimyeloma and bone-protective effects on myeloma cells and preclinical models. To the best of our knowledge, this compound is the first HDACi that was independently developed in China and shows both anti-myeloma and anti-osteolytic bone disease activity in vitro and in vivo, supporting the future clinical application of this drug as a treatment for MM.

\section{Methods}

\section{Drugs}

Chidamide was a generous gift from the Chipscreen Company, Shenzhen, China. Additional information regarding drugs and reagents is provided in the Online Supplementary Materials.

\section{Human myeloma cell lines, primary myeloma samples, bone marrow stromal cells (BMSCs), healthy peripheral blood mononuclear cells (PBMCs) and bone marrow-derived mononuclear cells (BMMCs)}

The human myeloma cell lines RPMI-8226, MM.1s, and U266B1 were purchased from the cell bank of the Chinese Academy of Science; H929, CAG, ARP-1, LP-1, and OPM2 cells were generous gifts from Dr. Oing Yi (Department of Cancer Biology, Lerner Research Institute, Cleveland Clinic, Cleveland, OH, USA). ANBL-6 cells were a generous gift from Professor Zhiqiang Liu, Tianjin Medical University, China. The luciferaseexpressing myeloma cell line RPMI-8226-luc was constructed by infecting RPMI-8226 cells with a lentivirus encoding the luciferase gene, and infected cells were selected with puromycin. Primary myeloma samples and BMSCs were collected from newly diagnosed patients, and PBMCs and BMMCs were collected from healthy donors. Primary CD138(+) cells were sorted using CD138 microbeads (Miltenyi Biotech, CA, USA).

\section{Cell culture}

Myeloma cell lines were cultured in RPMI 1640 medium supplemented with $10 \%$ fetal bovine serum and $1 \%$ L-glutamine at $37^{\circ} \mathrm{C}$ in a $5 \% \mathrm{CO}_{2}$ atmosphere. BMSCs were cultured in minimum essential medium $\alpha$ (MEM- $\alpha$ ) supplemented with $10 \%$ fetal bovine serum and $1 \%$ L-glutamine. BMSCs were cultured as premature osteoblasts for seven days and as mature osteoblasts for 21 days in the human mesenchymal stem cell (MSC) osteogenic differentiation basal medium.

\section{Cell proliferation, cell cycle, and cell apoptosis assays}

Cell counting kit-8 (CCK-8) assays were used to detect MM cell viability. Flow cytometry was used to assess the cell cycle and apoptosis. A detailed description of each procedure is provided in the Online Supplementary Materials.

\section{In vitro $\mathrm{OC}$ differentiation, pit formation and F-actin ring formation}

Osteoclasts were differentiated from PBMCs in osteoclastogenic medium as previously described. ${ }^{12}$ Briefly, MEM- $\alpha$ contain- ing $50 \mathrm{ng} / \mathrm{ml}$ RANKL and $25 \mathrm{ng} / \mathrm{ml}$ monocyte colony stimulating factor (M-CSF) supplemented with 10\% fetal bovine serum and $1 \%$ L-glutamine was used as osteoclastogenic medium for cells cultured in the presence or absence of chidamide. PBMCs from healthy donors were cultured as pre-OCs for 14 days and as mature OCs for 21 days. The F-actin ring formation assay, assessment of the resorption ability of OCs and tartrate-resistant acid phosphatase $(\mathrm{TRAP})^{+}$staining were performed as described in a previous report. $^{12}$

\section{Drug treatments in different mouse models}

Two tumor-bearing murine models and a non-tumor-bearing model were employed to investigate the anti-tumor and bone-protective effects of chidamide. ${ }^{4,13-15}$ Micro computed tomography (CT) was used to evaluate MM bone disease. Further details are provided in the Online Supplementary Materials. ${ }^{16,17}$

\section{Statistical analysis}

All data are expressed as means \pm SEM and are representative of at least three experiments with similar results performed in triplicate, unless indicated otherwise. A two-tailed Student's $t$-test was used to determine the significance of differences between two groups, and one-way analysis of variance was used to estimate the differences between three or more groups. GraphPad Prism 5.0 software (GraphPad Software, CA, USA) was used for the analysis.

\section{Ethical approval}

Primary myeloma samples, BMSCs, PBMCs, and BMMCs were collected after obtaining informed consent from the patients and approval from the Ethics Committee of the First Affiliated Hospital of Zhejiang University. The animal experiments were carried out after obtaining approval from the Ethics Committee of the First Affiliated Hospital of Zhejiang University.

\section{Results}

\section{Chidamide exhibits HDAC inhibitory activity in MM cell lines}

We first investigated the basal expression levels of HDAC1, 2, 3, and 10, which are chidamide targets, in MM cell lines. As shown in Figure 1A, HDAC1, HDAC2 and HDAC3 were all expressed in the nine myeloma cell lines (H929, LP-1, ARP-1, U266B1, RPMI-8226, ANBL-6, OPM2, CAG, and MM.1s), and both HDAC2 and HDAC3 were expressed at slightly higher levels than HDAC1. HDAC10 was barely detectable in human myeloma cell lines. Next, we measured HDAC activity and the acetylation of lysine residues on histones $\mathrm{H} 3$ and $\mathrm{H} 4$ to determine the inhibitory effect of chidamide on HDAC. As shown in Figure 1B,C, chidamide inhibited HDAC activity and significantly increased the acetylation of $\mathrm{H} 3 \mathrm{~K} 8$, H3K18 and H4K8. However, the expression of HDAC1, HDAC2, HDAC3 and HDAC10 was not affected (Figure 1D).

\section{Chidamide not only exerts anti-myeloma effects but also overcomes the resistance conferred by the BM microenvironment}

Subsequently, we evaluated the anti-myeloma effect of chidamide. Cell lines were treated with increasing concentrations of chidamide $(0.5-8 \mu \mathrm{M})$ for different times $(24,48$, or $72 \mathrm{~h}$ ). CCK-8 assays revealed a dose-dependent and time-dependent pattern of chidamide cytotoxicity (Online Supplementary Figure S1A). Flow cytometry (Annexin V 
A

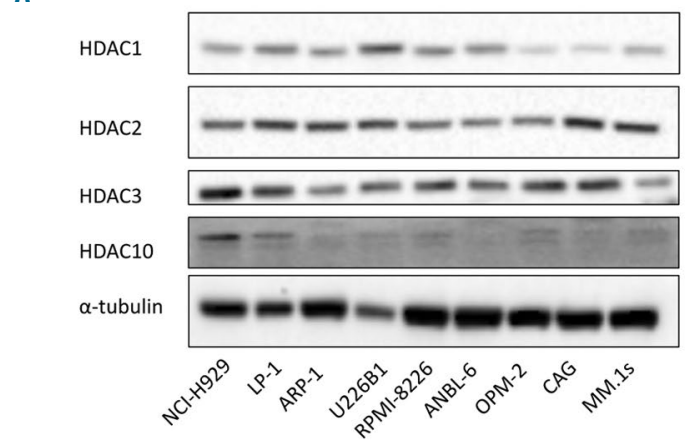

B $\quad$ HDAC activity (\% of control)

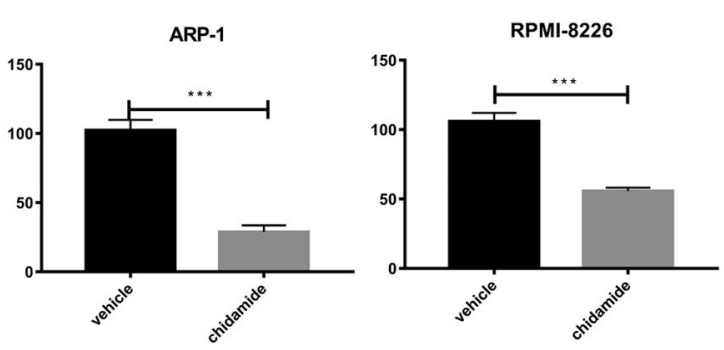

D

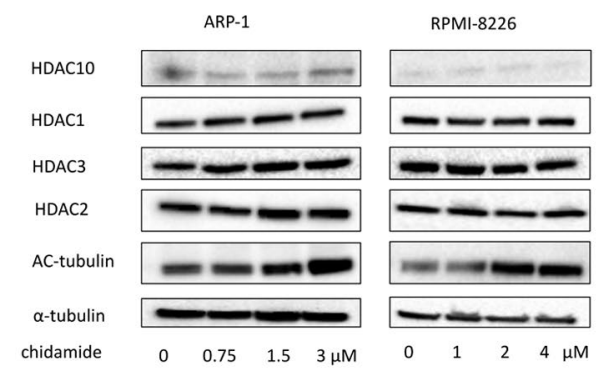

Figure 1. Chidamide exhibits HDAC inhibitory activity in MM cell lines. (A) Western blotting was used to detect the protein levels of HDAC1, HDAC2, HDAC3 and HDAC10. (B) HDAC activity assay showed that chidamide could significantly inhibit HDAC activity (Both in ARP-1 and RPMI-8226, $* * * P<0.001$ ). (C) Western blot analysis of $\mathrm{H} 3$ acetylation at Lys 18 and Lys9 and $\mathrm{H} 4$ acetylation at Lys8. H3 and H4 expression levels were used as loading controls. (D) Western blot analysis of HDAC1, HDAC2, HDAC3, and HDAC10 levels. Acetylation of $\alpha$-tubulin at K40 was also examined, and $\alpha$-tubulin expression was used as the loading control. HDAC: histone deacetylase.

and propidium iodide [PI] staining) showed that chidamide significantly induced MM cell apoptosis at $48 \mathrm{~h}$ and $72 \mathrm{~h}$ (Figure $2 \mathrm{~A}$ and Online Supplementary Figure $S 1 B$ ). ARP-1, MM.1s and CAG cells were more sensitive to chidamide, with $\mathrm{IC}_{50}$ values of approximately $2-4 \mu \mathrm{M}$, whereas the $\mathrm{IC}_{50}$ values were approximately $4-6 \mu \mathrm{M}$ for the other six cell lines at $48 \mathrm{~h}$. When the incubation time was extended to $72 \mathrm{~h}$, the $\mathrm{IC}_{50}$ values of most of the cell lines were 1.5-4 $\mu \mathrm{M}$. Furthermore, incubation of ARP-1 and MM.1s cells with an optimal concentration of chidamide increased the levels of cleaved caspases 3, 7, 8, and 9 and cleaved PARP-1 cleavage (Figure 2B). To evaluate whether apoptosis was induced in a caspase-dependent or caspaseindependent manner, we incubated MM.1s and ARP-1 cells with chidamide in the presence of a pan-caspase inhibitor, Q-VD-Oph, for 48h. After co-incubating chidamide with Q-VD-Oph, the apoptotic cells were significantly reduced (Figure 2C and Online Supplementary Figure $2 A)$, with down-regulated active caspase- 3 and caspase- 9 expression (Online Supplementary Figure 3). Additionally, the cell cycle arrest effect of chidamide was evaluated by flow cytometry. ARP-1, OPM-2, LP-1, and RPMI-8226 cells were treated with increasing doses of chidamide $(0.25-6 \mu \mathrm{M})$ for $48 \mathrm{~h}$; chidamide induced ARP-1 cell cycle arrest in the G0/G1 phase and reduced the percentage of cells in the proliferation phases (S and G2) regardless of co-incubation with $\mathrm{Q}-\mathrm{VD}-\mathrm{Oph}\left({ }^{* * *} P<0.001\right.$, Figure $\left.2 \mathrm{D}\right)$. OPM-2, RPMI-8226, and LP-1 cells showed similar results after incubation with chidamide (Online Supplementary Figure 4).

In addition, as shown in Figure 2E, chidamide induced the apoptosis of CD138-positive cells from patients with $\mathrm{MM}(\mathrm{n}=5,48 \mathrm{~h})$. Moreover, the BMMCs from four healthy donors were incubated with $4 \mu \mathrm{M}$ chidamide for $48 \mathrm{~h}$ to examine the cytotoxicity of chidamide toward non-tumor cells. Although approximately $10 \%$ of monocytes underwent apoptosis, the numbers of apoptosis cells were only slightly higher than the vehicle group and far less than MM cells (Online Supplementary Figure 2B).

Furthermore, we investigated the cytotoxicity of chidamide in the presence of the BM microenvironment. For this purpose, H929 cells were co-cultured with BMSCs and OCs in the presence of $6 \mu \mathrm{M}$ chidamide for $48 \mathrm{~h}$. OCs showed a significant anti-apoptotic effect, but pretreatment with chidamide during osteoclastogenesis suppressed the protective effect of OCs on myeloma cell apoptosis (Figure 3A, Figure 3C). Chidamide-induced apoptosis in approximately $30 \%$ of the MM cells, even after co-culture with BMSCs, which usually exert a protective effect on MM cells (Figure 3B,C).

\section{The molecular mechanisms underlying chidamide activity in myeloma cells}

When ARP-1 and RPMI-8226 cells were treated with an increasing dose of chidamide $(0-6 \mu \mathrm{M})$ (Figure $4 \mathrm{~A}$ ), the expression of Myc, Mcl-1, and Bcl-xL decreased in a dosedependent manner. Additionally, in the presence of chidamide, levels of cell cycle-related proteins, including p27, p21, Cyclin-D2, CDK4, and CDK6, were all reduced (Figure 4A). Finally, as shown in Figure 4B, SOCS3 levels increased and p-JAK2 and p-STAT3 levels decreased in RPMI-8226 and ARP-1 cells treated with different concentrations of chidamide. Additionally, a pan-caspase inhibitor, Q-VD-Oph, was used to treat MM cells together with chidamide to prevent inhibitory effects of activated caspases on the levels of signaling and apoptosis-related 
proteins. As shown in Figure 4 and Online Supplementary Figure S5, most of these proteins showed the same changes in the presence and absence of Q-VD-Oph.

\section{Chidamide decreases the MM tumor burden and prevents myeloma-associated bone loss in a mouse model of disseminated myeloma}

Two murine models were employed to examine the dual anti-tumor and bone-protective effects of chidamide. First, as shown in Figure 5A, a significant decrease in tumor volume was observed in chidamide-treated NOD/SCID mice compared with that observed in the vehicle group. Consistent with the Western blot results from the cell lines, immunohistochemical staining revealed decreased expression of Bcl-xL, CDK4, CDK6 and Myc and increased levels of cleaved caspase-3 (Online
Supplementary Figure S6). Next, NCG mice were intravenously injected with RPMI-8226-luc cells to establish the murine model of myeloma-induced bone destruction. Compared with the vehicle group, chidamide clearly controlled the tumor burden, as measured by serum levels of human Ig $\lambda$ light chain secreted by RPMI-8226-luc cells ${ }^{* * *} P<0.001$, Figure $\left.5 \mathrm{~B}\right)$ and the bioluminescence in vivo imaging (Figure $5 \mathrm{~B}$ ). Then, serum levels of the bone resorption marker Carboxy-terminal telopeptide 1 (CTXI) were measured and were found to be significantly diminished in chidamide-treated mice ${ }^{* *} P<0.01$, Figure 5C); in addition, a significantly increased serum level of amino-terminal propeptide (PINP), the bone formation marker, was observed ( ${ }^{* * *} P<0.001$, Figure $5 \mathrm{C}$ ). Consistent with these findings, micro-CT three-dimensional reconstructed images at the metaphysis of distal femurs
A
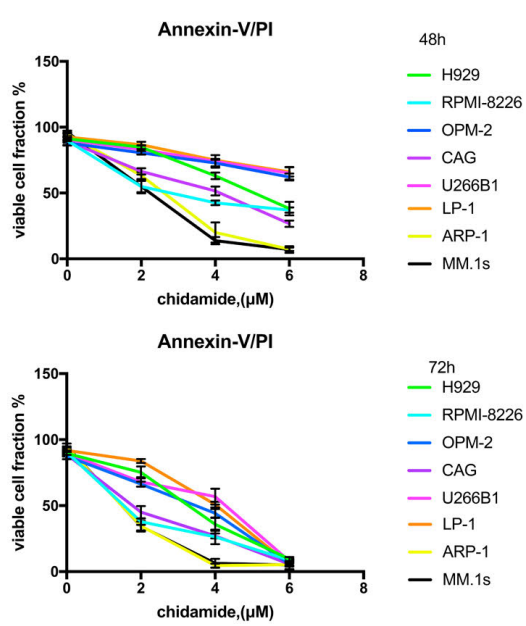

C

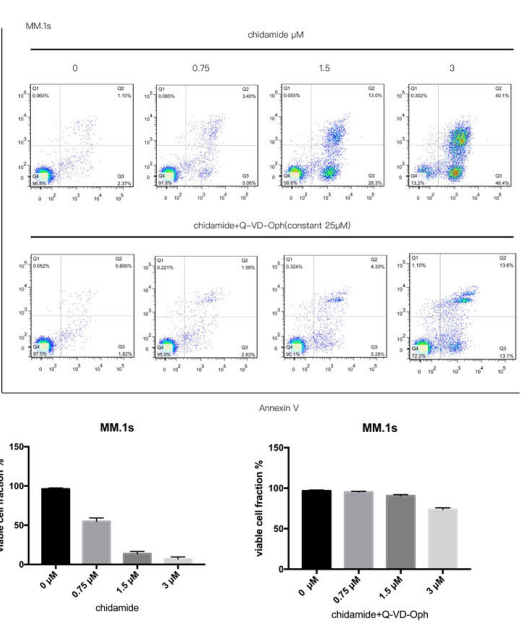

primary MM cells (CD138+)

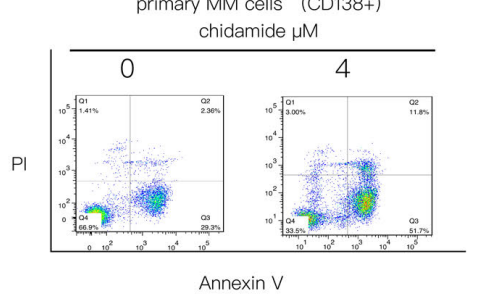

B
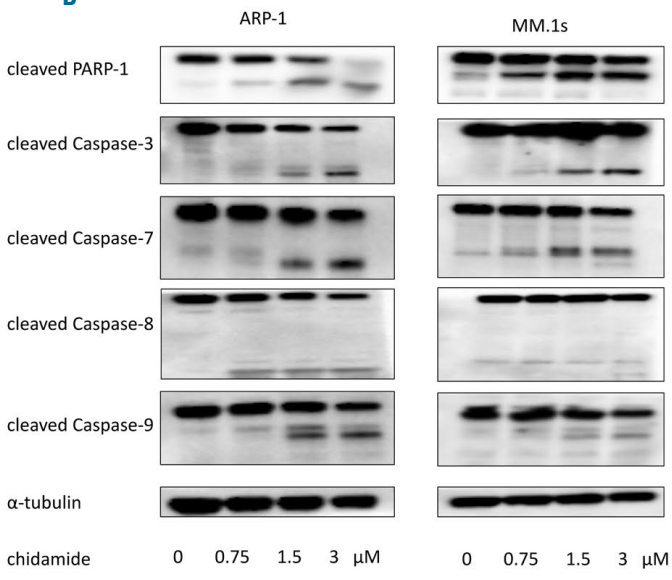

D
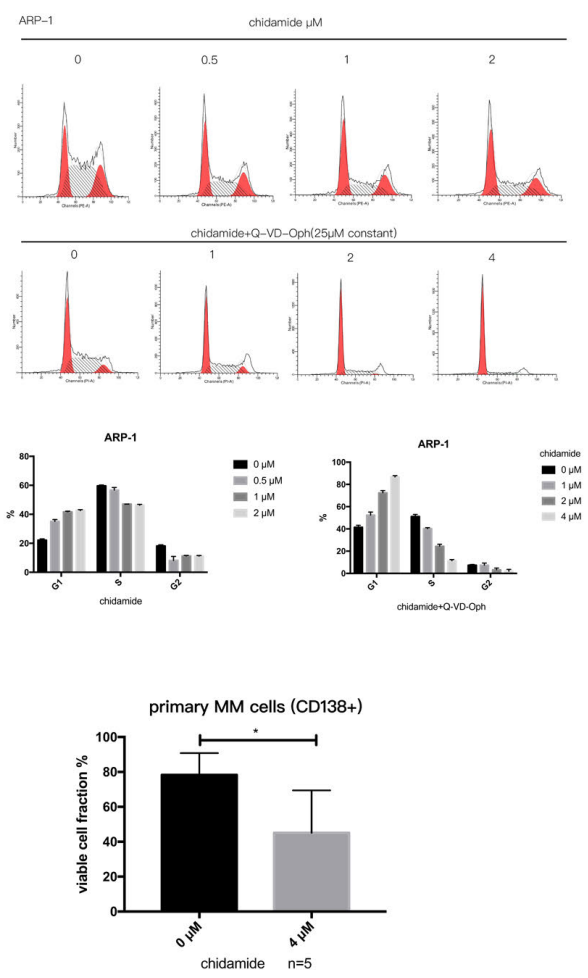

Figure 2. MM cells are vulnerable to chidamide. (A) The cytotoxicity of chidamide toward MM cell lines was examined using flow cytometry (Annexin V/PI), and all data are summarized as the means \pm SEM $(n=3)$. (B) The expression of apoptosis-associated proteins, including PARP-1 and cleaved caspases $3,7,8$, and 9 , in chidamide-treated ARP-1 and MM.1s cells was detected. (C) The effect of chidamide on apoptosis in MM.1s cells after $48 \mathrm{~h}$ with or without coincubation with the pan-caspase inhibitor Q-VD-Oph. After co-incubation with Q-VD-Oph, chidamide-induced apoptosis was inhibited. (D) Cell cycle arrest was examined using flow cytometry, and ARP-1 cells underwent G1 phase arrest in response to incubation with chidamide regardless of co-incubation with QVD-Oph. (E) Chidamide could significantly induce + primary CD138 cell apoptosis after incubation for $48 \mathrm{~h}$ ( $\mathrm{n}=5$, $\left.{ }^{*} P<0.05\right)$. (E) Chidamide could significantly induce primary $\mathrm{CD} 138^{+}$cell apoptosis after incubation for $48 \mathrm{~h}$ $(n=5)$. PI: propidium iodide; MM: multiple myeloma. 
revealed greater myeloma-associated bone loss in the vehicle group as compared with the chidamide-treated group (Figure 5D). Moreover, based on the bone morphometric parameters, chidamide increased the trabecular number $\left({ }^{* *} P<0.01\right)$ and bone volume density $(P=0.0755)$ and reduced trabecular separation $\left({ }^{* *} P<0.01\right)$ compared to the control group (Figure 5E).

\section{Chidamide inhibits osteoclastogenesis and bone resorption in vitro}

As the in vivo study revealed that chidamide exerts antimyeloma effects, we investigated the effect of chidamide on the formation and function of OCs of human origin. The expression of DUSP1, c-fos, NFATc1 and HDAC10 increased during RANKL-induced OC formation (Figure 6A). We then tested several key factors and signaling path- ways that mediate osteoclastogenesis in order to identify the mechanisms underlying the aforementioned effects. The levels of p-p38, p-ERK1/2, p-JNK and p-AKT were also reduced, indicating that chidamide suppressed the classical pathways of OC activation. Cathepsin K, c-fos, HDAC10 and NFATc1 expression levels were all downregulated after chidamide treatment in a dose-dependent manner. In addition, acetylation of H3K9, H3K18 and H4K8 was increased after chidamide treatment (Figure 6B). PBMCs cultured in osteoclastogenic medium were incubated with different concentrations of chidamide to evaluate its effects on $\mathrm{OC}$ formation. At a low concentration $(0-1 \mu \mathrm{M})$ of the drug, the number of TRAP+ multinucleated cells derived from healthy donors was reduced, but the density of cells was not significantly reduced $\left({ }^{* *} P<0.01\right.$, Figure 6C,D). Additionally, F-actin ring forma-
A

$\mathrm{Pl}$

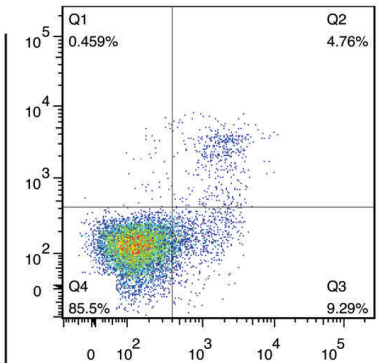

H929

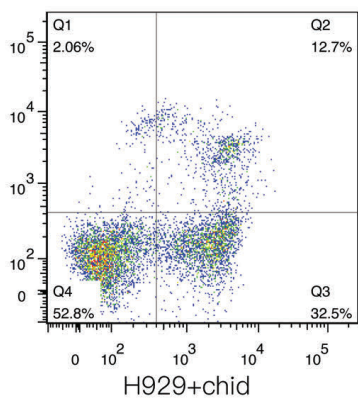
H929+chid

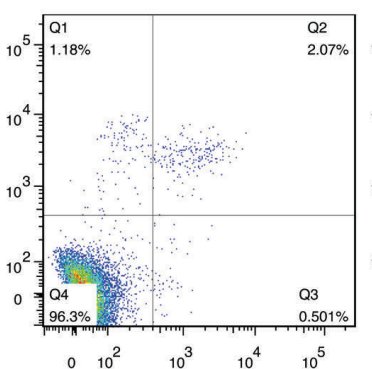

$\mathrm{H} 929+\mathrm{OCS}$

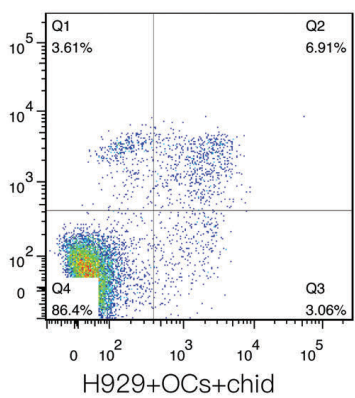

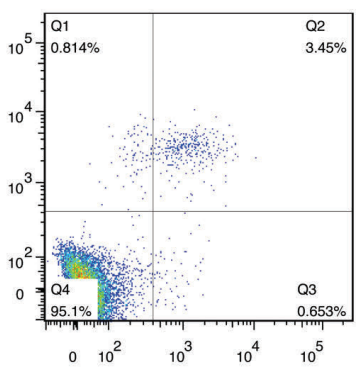

$\mathrm{H} 929+\mathrm{OCs}$ (chid)

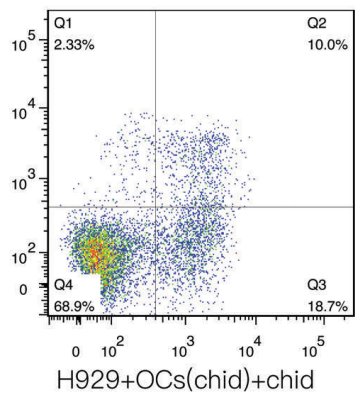

Q3

B

Annexin V

$\mathrm{Pl}$

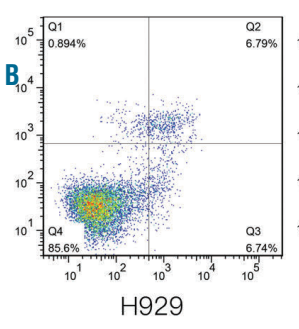

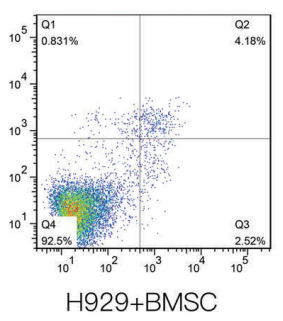

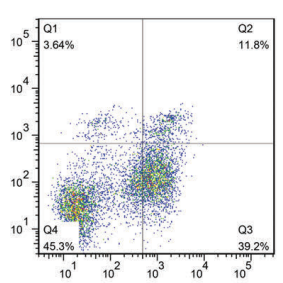

H929+chid

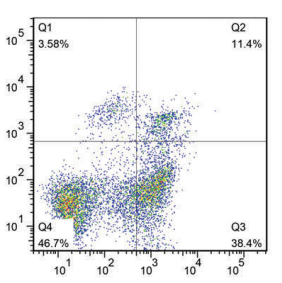

H929+BMSC+chid
C

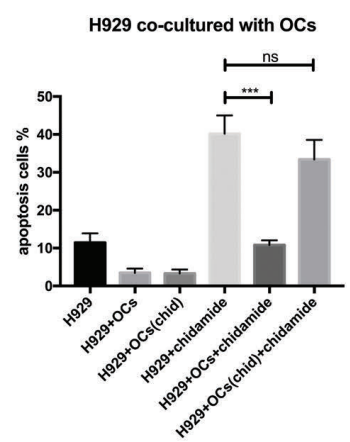

Annexin V

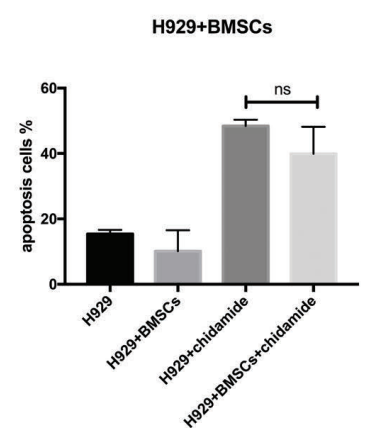

Figure 3. Chidamide overcame the resistance conferred by chidamide-pretreated OCs and BMSCs. (A) and (C) H929 cells were co-cultured with OCs and chidamide-pretreated OCs for $48 \mathrm{~h}$ or with $6 \mu \mathrm{M}$ chidamide, and cell apoptosis was detected by flow cytometry after staining with Annexin V and PI. (B) and (C) H929 cells were co-cultured with BMSCs or with $6 \mu \mathrm{M}$ chidamide for $48 \mathrm{~h}$, and cell apoptosis was detected by flow cytometry after staining with Annexin $\mathrm{V}$ and $\mathrm{PI}(\mathrm{H} 929+$ chidamide vs. H929+OCs+chidamide, $* * * P<0.001$; H929+chidamide vs. H929+OCs (chida)+chidamide $P>0.05$, non-significant; H929+chidamide vs. H929+BMSCs+chidamide, $P>0.05$ non-significant). PI: propidium iodide; OC: osteoclasts; BMSC: bone marrow stromal cell. 
tion was inhibited in the presence of chidamide during osteoclastogenesis (Figure 6C). Next, we evaluated functional changes in OCs cultured on calcium substrate-coated slides. Chidamide treatment induced a dose-dependent reduction in the area of resorption pits. With increasing drug doses, the resorption area was substantially reduced (Figure 6C, Figure $6 \mathrm{E}^{* * *} P<0.001$ ).

\section{Effect of chidamide on osteoblasts}

HDAC1 and HDAC3 are regarded as negative regulators of osteoblastogenesis during bone formation, thus we also evaluated the effect of chidamide on osteoblasts. As shown in Online Supplementary Figure S7, primary BMSCs from patients with $M M(n=6)$ were cultured in human MSC osteogenic differentiation basal medium in the presence of different chidamide concentrations. Alkaline phosphatase (ALP) was measured as a positive marker of osteoblast differentiation, and its expression was slightly increased by chidamide treatment. Additionally, Alizarin red staining (ARS) of calcium deposits showed that chidamide had no promotion or inhibitory effect on osteoblast differentiation (Online Supplementary Figure $S 7 A, B)$. Finally, the expression of osteocalcin $(O C N)$, Activin $A$ and semaphorins at the messenger ribonucleic acid (mRNA) level was also examined; OCN (day 21) was increased, while Activin A (day 21) was down-regulated (Online Supplementary Figure S7C).

\section{Chidamide exerts bone-protective effects on non-tumor-bearing mice}

Chidamide was administered via oral gavage to nontumor-bearing C57BL $/ 6$ mice at a dose of $25 \mathrm{mg} / \mathrm{kg}$ for 21 days to establish whether chidamide directly exerts its bone-preserving effect on the bone tissue or exerts an indirect effect by decreasing the tumor burden. As shown in Figure 7A, serum CTX-I levels were not significantly different between the vehicle $(n=5)$ and chidamide $(n=5)$ groups, whereas the serum PINP level was clearly increased after treatment with chidamide $\left.{ }^{* * *} P<0.001\right)$. Thereafter, in vivo intraperitoneal injections of soluble receptor activator of nuclear factor- $\mathrm{KB}$ ligand (sRANKL; three doses) were administered within $50 \mathrm{~h}$ followed by gavage with chidamide for 21 days to mimic OC stimula- tion by myeloma-derived sRANKL. Serum CTX-I levels increased in both the vehicle group $(n=5)$ and the chidamide group $(n=5)$ after sRANKL injections, but the level in the chidamide-treated group was increased to a lesser extent than the level in the vehicle group $\left({ }^{*} P<0.05\right)$. Serum PINP levels were reduced in both groups; however, chidamide attenuated the reduced PINP level in the chidamide-treated group $\left({ }^{* * *} P<0.001\right)$. TRAP+ staining of bone showed a reduced number of OCs (Figure 7B,C) in the chidamide-treated group $\left({ }^{* * *} P<0.001\right)$. Moreover, the bone morphometric parameters evaluated by micro-CT (Figure 7D,E) indicated that chidamide increased the trabecular number $\left.{ }^{* * *} P<0.001\right)$ and reduced trabecular separation compared to the control group $\left.{ }^{* *} P<0.01\right)$. Although bone volume density over total volume (BV/TV, $P>0.05$ ) showed an increasing trend in the chidamide-treated group, the difference between the two groups was not significant. Based on these results, chidamide increased the bone volume of healthy mice to some extent and directly prevented RANKL-induced OC activation.

\section{Discussion}

HDACs are an important family of enzymes with crucial roles in carcinogenesis through their repressive effects on tumor suppressor gene transcription and are proposed as therapeutic targets in oncology. ${ }^{18,19}$ HDAC inhibitors induce cancer cell apoptosis, cell cycle arrest and promote differentiation, particularly in hematological malignancies. However, the anti-cancer effects of HDAC inhibitors differ and their pharmacological effects vary, depending on the cancer cell types, HDAC targets and doses. Chidamide, a novel HDACi which is currently being widely used in China to treat patients with T-cell lymphoma, shows good efficacy and tolerability. ${ }^{5}$ In our investigation herein, we evaluated and explored the efficacy of chidamide in treating myeloma and its associated osteolytic bone disease.

Chidamide reduced myeloma cell viability in both primary MM cells and MM cell lines, even in the presence of BMSCs or chidamide-pretreated OCs. As the microenvironment is crucial for myeloma drug resistance and
A

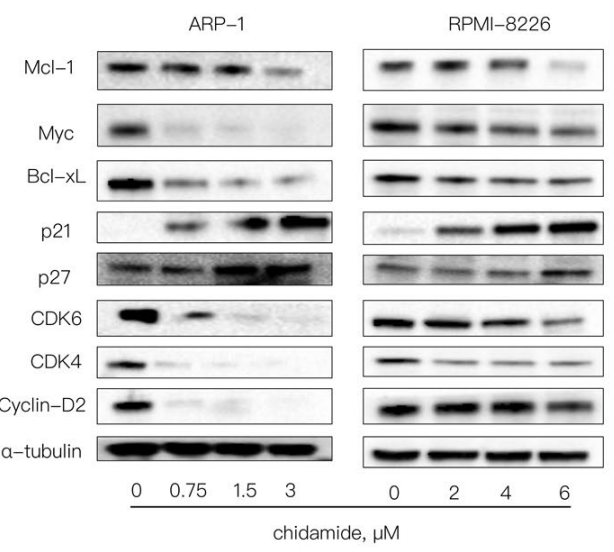

B

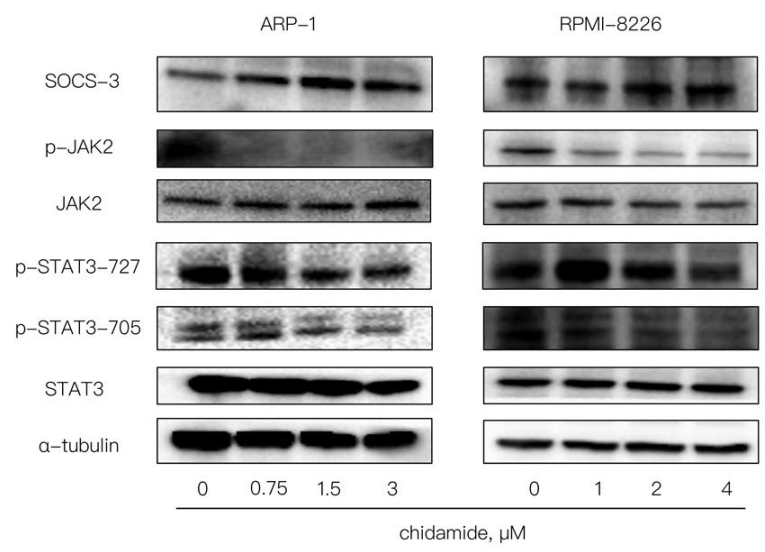

Figure 4. The molecular mechanisms underlying chidamide activity in myeloma cells. (A) Western blot analysis of Mcl-1, Myc, Bcl-xL, Bcl-2, p21, p27, CDK4, CDK6, and Cyclin-D2 levels; $\alpha$-tubulin was used as the loading control. (B) SOCS-3, p-JAK2, JAK2, p-STAT3-727, p-STAT3-705, and STAT3 levels were analyzed by Western blotting; $\alpha$-tubulin was used as the loading control. 
relapse, our data has shown that chidamide overcomes the drug resistance mediated by the interaction between MM cells and the microenvironment. Moreover, the good anti-myeloma effect of chidamide on the disseminated MM mouse model reveals the efficacy of chidamide in the presence of the BM microenvironment.

HDACs regulate a variety of targets involved in differ- ent cell functions and processes, such as apoptosis, the cell cycle and differentiation. ${ }^{20}$ Chidamide inhibited HDAC activity and induced cell cycle arrest in G1 phase, accompanied by cell apoptosis. Notably, cell cycle arrest and the alterations in levels of related proteins were not reversed by the pan-caspase inhibitor. HDAC inhibitors cause cell cycle arrest though several pathways, the most important
A

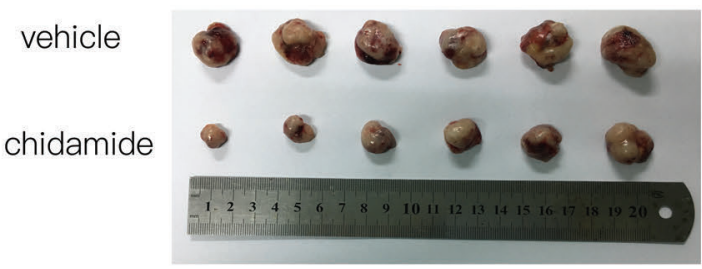

B day 14
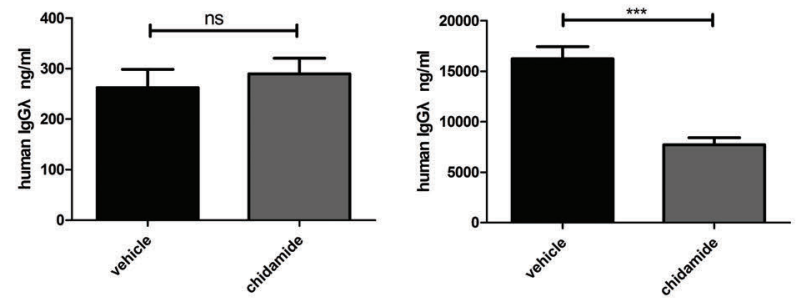

day 28

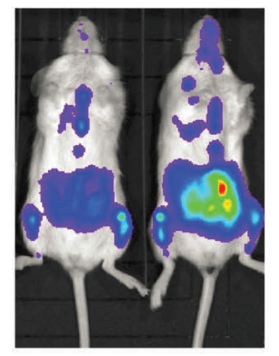

vehicle

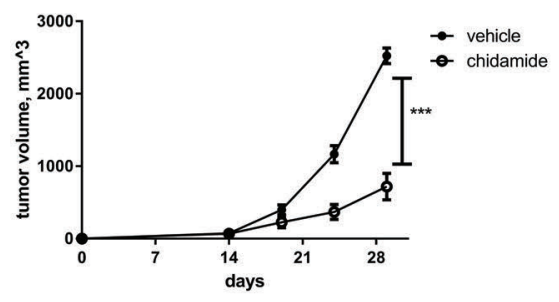

day 28

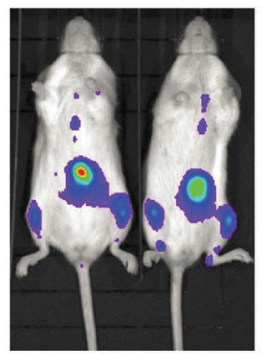

chidamide

$c$

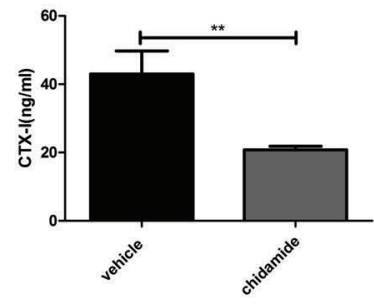

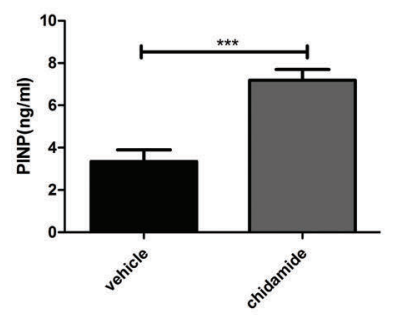

D

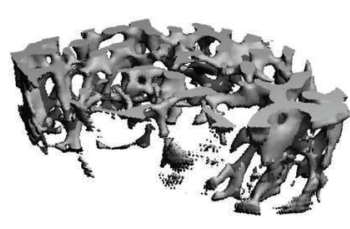

vehicle

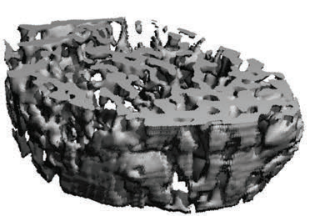

chidamide

$E$
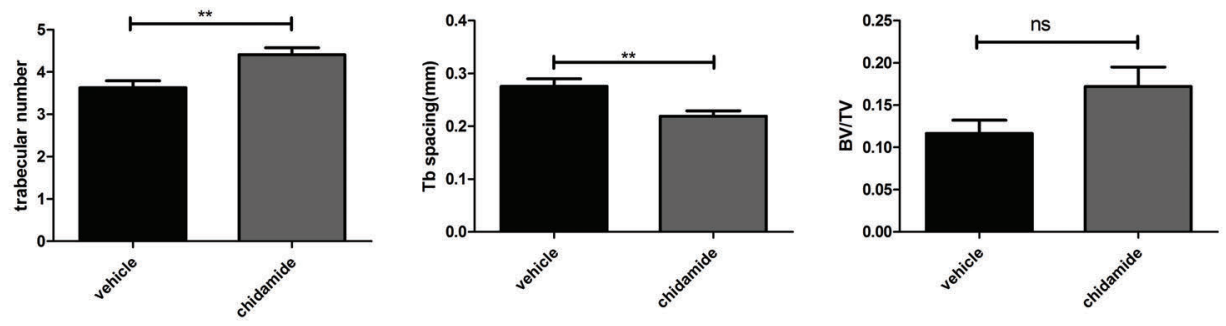

Figure 5. Dual anti-myeloma and bone-protective effects of chidamide on a disseminated myeloma murine model. (A) Chidamide suppresses the growth of MM xenografts, $* * * P<0.001$. (B) Tumor volumes, serum human lg $\lambda$ levels in each group and in vivo bioluminescence imaging were used to compare the tumor burdens between the two groups. (C) Serum levels of CTX-I $(* * P<0.01)$ and PINP $(* * * P<0.001)$ in each group. (D) Micro-CT three-dimensional reconstructed images from the vehicle and chidamide groups. (E) Micro-CT parameters of the bone in the vehicle and chidamide groups. Trabecular numbers, ***P<0.001; trabecular space, $* * P<0.01 ; \mathrm{BV} / \mathrm{TV}$, non-significant (ns). 
of which seems to be increasing the expression of cell cycle genes. p21 plays a crucial role in chidamide-induced G1 cell cycle arrest. HDAC1 regulates p21 expression to some extent. HDAC1 interacts with the p 21 promoter by competing with the p53 protein to decrease the transcription of $\mathrm{p} 21 .{ }^{21}$ Based on our results from MM cell lines, in chidamide-treated MM cells HDAC1 was released from the $\mathrm{p} 21$ promotor, subsequently increasing p21 expression. Additionally, chidamide inhibited the expression of the Cyclin-D2 gene, thus inhibiting the functions of CDK4 and CDK6. Aberrant activation of the JAK2/STAT3 pathway is required for the pathogenesis of a variety of cancers..$^{22,23}$ Inappropriate STAT3 activation protects cells from apoptosis. HDAC3 targets STAT3, and inhibition of HDAC3
A

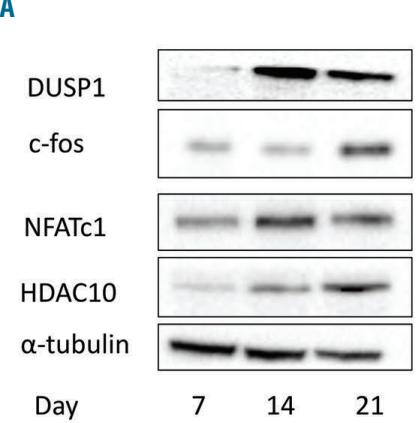

B

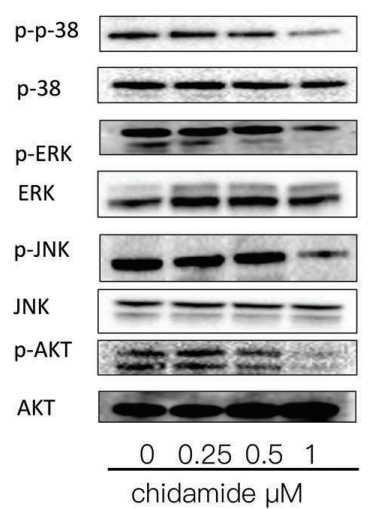

C

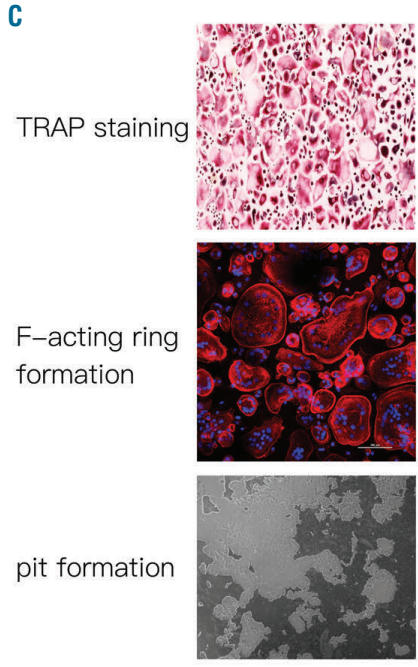

0
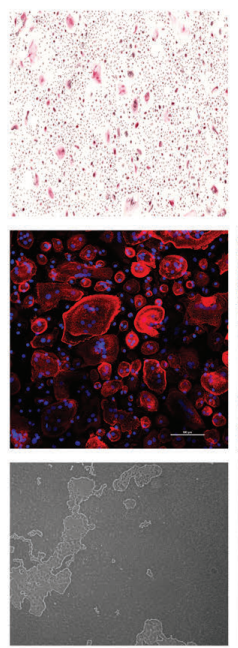

0.25
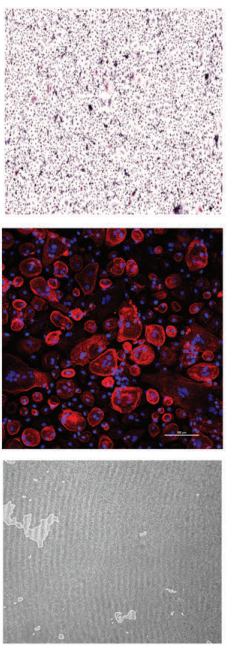

0.5
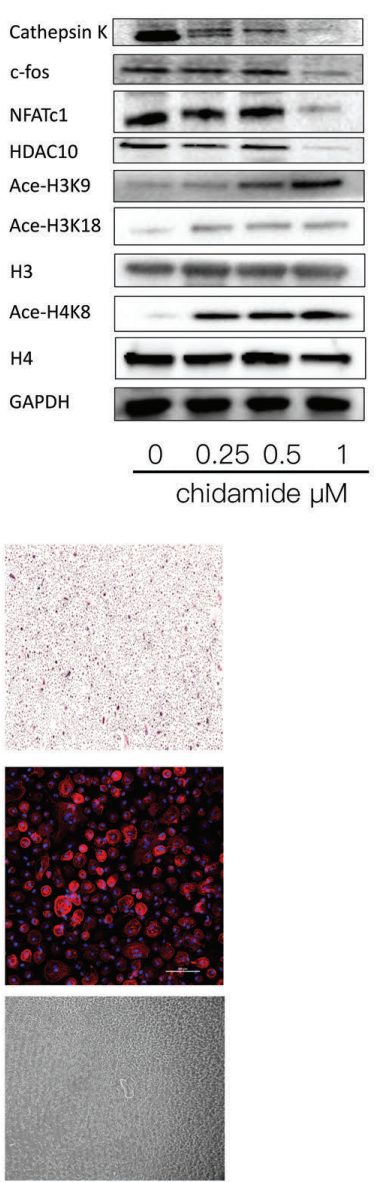

1

chidamide $\mu \mathrm{M}$

D

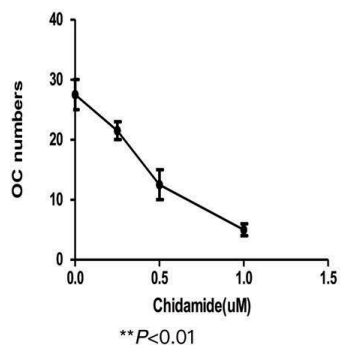

E

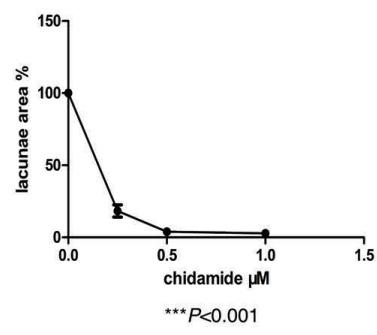

Figure 6. Chidamide inhibited osteoclast differentiation in vitro. (A) Western blotting was used to detect the expression levels of key factors (DUSP1, c-fos, NFATc1, and HDAC10) during OC maturation; $\alpha$-tubulin was used as the loading control. (B) Western blot analysis of the expression levels of pathway proteins including $p$ p38, p38, p-ERK, ERK, p-JNK, JNK p-AKT, and AKT; levels of key factors, including Cathepsin K, c-fos, NFATc1 and HDAC10, in OCs treated with different concentrations of chidamide. GAPDH was used as the loading control. Acetylation of H3K18 and H4K8 was also evaluated; H3 and H4 were used as loading controls. (C) TRAP staining of OCs treated with different concentrations of chidamide. F-actin ring formation. Rhodamine-conjugated phalloidin was used to visualize F-actin (red), and nuclei were stained with DAPI (blue), 200×. Pit formation assay of OCs treated with different concentrations of chidamide. (D) The number of TRAP-positive cells was reduced in cultures treated with increasing doses of chidamide, $* \star P<0.01$. (E) Calcium resorption was reduced in the presence of chidamide $(0-1 \mu \mathrm{M})$. Resorption in the lacunae area (\%) diminished with increasing doses of chidamide, $* \star * P<0.001$. OC: osteoclasts; TRAP: tartrate-resistant acid phosphatase. 
A
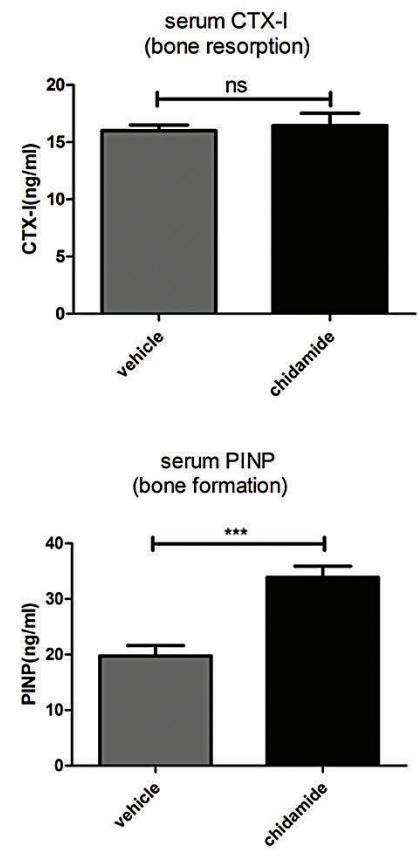

serum CTX-I
(bone resorption,following in vivo RANKL)

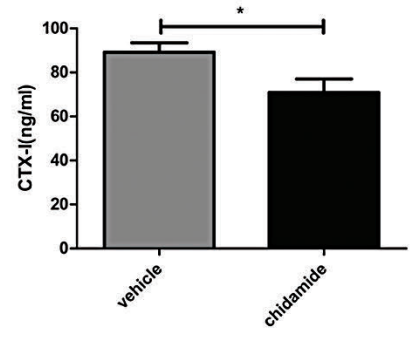

serum PINP

(bone formation,following in vivo RANKL)

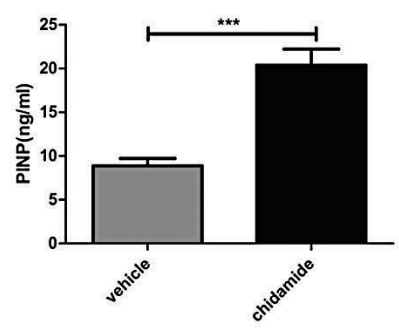

B

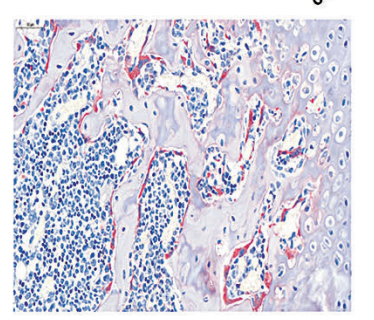

vehicle

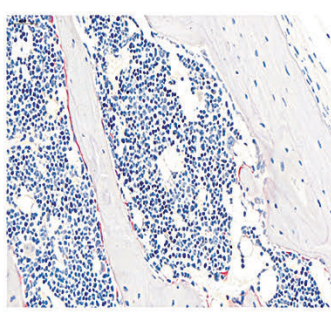

chidamide

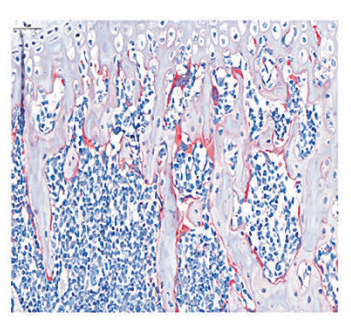

vehicle+RANKL

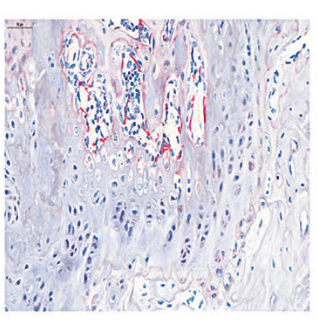

chidamide+RANKL
C
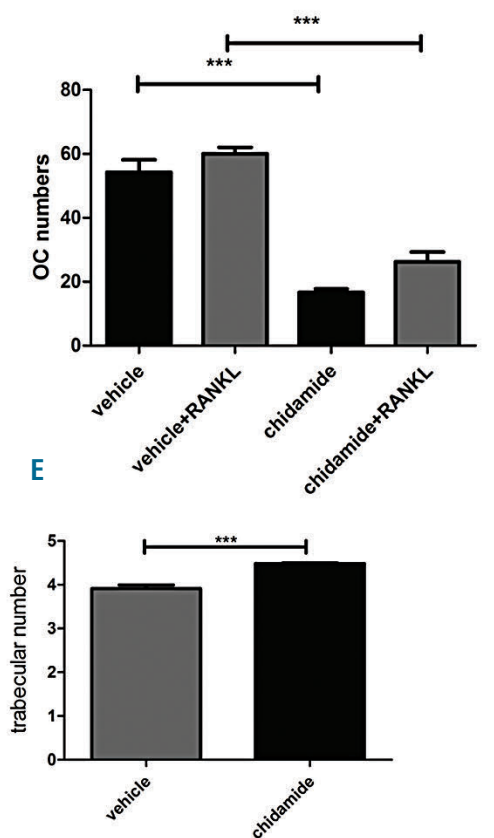

D

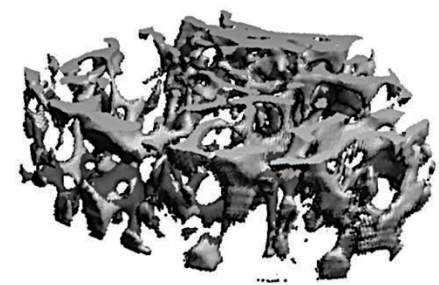

vehicle

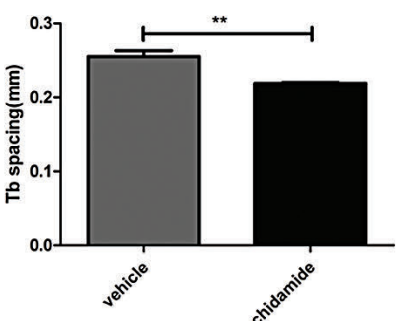

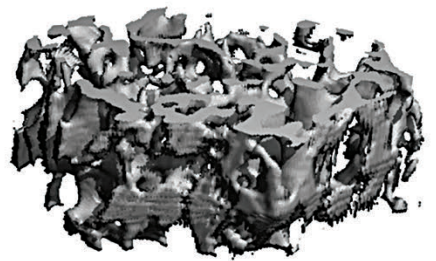

chidamide

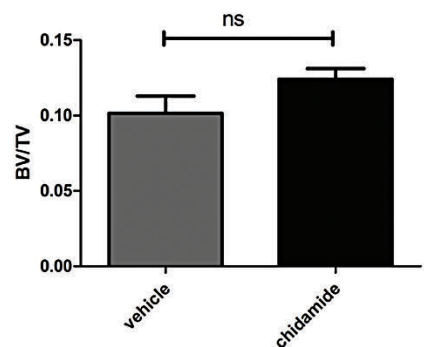

Figure 7. Chidamide inhibited osteoclast differentiation and the function of chidamide in non-tumor-bearing mice was examined. (A) Serum CTX-I and PINP levels before (CTX-1, P>0.05, non-significant; PINP, $* * * P<0.001)$ and after intraperitoneal injections of sRANKL following chidamide treatment (CTX-1, $* P<0.05$; PINP, $* * * P<0.001$ ). (B) TRAP staining of mouse femur bone tissues. (C) Chidamide treatment decreased the number of OCs (TRAP-positive cells, dark red staining) in the mouse femur compared with the vehicle group; $* * * P<0.001$. (D) Micro-CT three-dimensional reconstructed images of the vehicle and chidamide groups. (E) MicroCT parameters of the bone in the vehicle and chidamide groups. Trabecular numbers, $* * * P<0.001$; trabecular space, $* * P<0.01$; BV/TV, non-significant (ns). RANKL: Receptor activator of nuclear factor K-B ligand; CTX: carboxy-terminal telopeptide; PINP: amino-terminal propeptide. 
induces STAT3 de-phosphorylation, thus reducing MM cell growth., Based on our results and findings from previous studies, we speculated that chidamide promoted MM cell apoptosis by inhibiting the JAK2/STAT3 signaling pathway. Furthermore, platelet factor 4 up-regulates SOSC3 expression to induce MM cell apoptosis by inhibiting STAT3. ${ }^{24}$ The expression of SOCS3, which acts as a tumor suppressor gene and is always silenced by epigenetic modulation in hematological malignancies, was up-regulated in chidamide-treated cells, which explains the decrease in p-JAK2 and p-STAT3 levels. ${ }^{2,25} \mathrm{Bcl}-2$ family members, including Bcl$\mathrm{xL}$ and Myc, are downstream targets of the JAK2/STAT3 signaling pathway. ${ }^{23}$ Moreover, active caspase- 3 is a negative regulator of the Bcl-2 family which represses the expression of Bcl-xL and Mcl-1 and subsequently induces cell apoptosis. ${ }^{26}$ Chidamide inhibited the constitutive activation of the JAK2/STAT3 pathway and caspase-3 activation, thus down-regulating Bcl-xL and Myc.

Myeloma-associated bone disease is a major complication of MM and decreases patients' quality of life. ${ }^{2,27}$ Interactions between myeloma cells and OCs not only support myeloma survival but also increase osteoclastogenesis and inhibit osteoblastogenesis, leading to osteolytic bone lesions and myeloma progression. ${ }^{28,29}$ An important finding of our study is that chidamide not only suppresses the formation and function of OCs in vitro, but also prevents myeloma-associated bone disease in vivo. Bone remodeling is a balance between bone resorption and bone formation. ${ }^{30}$ Active OCs, together with myeloma cells, increase bone resorption and inhibit osteoblast differentiation, leading to myeloma-associated bone disease. Chidamide-induced myeloma cell apoptosis prevented osteoclast maturation and showed no inhibitory effect on osteoblast differentiation. HDACs, which are targets of chidamide, are involved in OC formation. ${ }^{10,11,31}$ HDAC2 is reported to play an important role in OC maturation by activating the AKT pathway. ${ }^{11}$ Additionally, HDAC3 knockdown decreases the expression of NFATc1, Cathepsin K and DC-STAMP, thus inhibiting OC formation. ${ }^{31}$ Based on our data, chidamide repressed the expression of key factors, such as NFATc1, c-fos and Cathepsin K, during OC maturation, suggest- ing that chidamide suppresses OC differentiation by inhibiting the function of its targets (such as HDAC2 and HDAC3). A previous study reported the effect of HDAC10 on osteoclast differentiation. They showed that during OC differentiation, HDAC10 levels gradually increased, which is consistent with our study. However, when they knocked down HDAC10 in monocytes, OC differentiation was promoted, indicating that HDAC10 may have a negative effect on OC differentiation. ${ }^{32}$ However, in our study, following chidamide treatment and inhibition of OC formation, HDAC10 was downregulated at the protein level. Since chidamide can inhibit other HDACs in addition to HDAC10, its inhibitory effect on OC differentiation may not have been caused by HDAC10 down-regulation. Both MM cells and OCs can suppress the differentiation of osteoblasts. As a previous study reported, OCs inhibited osteoblast differentiation though exosomes containing micro ribonucleic acids (miRNAs) and some cytokines. ${ }^{33}$ In our study, chidamide could induce MM cell apoptosis and abrupt OCs maturation, indicating that chidamide may attenuate the inhibitory effect of MM cells and OCs on osteoblasts. When BMSCs were treated with chidamide during osteoblast differentiation, chidamide increased the gene expression levels of $A L P$ and $O C N$ while reducing the gene expression level of Activin $A$, which acted as a negative regulator in osteoblast differentiation via SMAD2mediated DLX5 down-regulation. ${ }^{34}$ The ARS experiment showed neither promotion nor an inhibitory effect on osteoblast differentiation. These results may explain the direct bone-protective effect of chidamide in the mouse models.

Our work reveals the dual anti-myeloma and bone-protective effects of chidamide in vitro and in vivo. These findings strongly support the potential clinical use of this drug as a treatment for $\mathrm{MM}$ in the near future.

\section{Funding}

This work was supported by the Funds for the National Natural Science Foundation of China (Grant No. 91742110 and 81471532) and the Funds for the Natural Science Foundation of Zhejiang province, China (LY17H080001).

\section{References}

1. Rollig C, Knop S, Bornhauser M. Multiple myeloma. Lancet. 2015;385(9983):21972208.

2. Silbermann R, Roodman GD. Myeloma bone disease: Pathophysiology and management. J Bone Oncol. 2013;2(2):59-69.

3. Christoulas D, Terpos E, Dimopoulos MA. Pathogenesis and management of myeloma bone disease. Expert Rev Hematol. 2009:2(4):385-398.

4. Gong K, Xie J, Yi H, Li W. CS055 (Chidamide/HBI-8000), a novel histone deacetylase inhibitor, induces G1 arrest, ROS-dependent apoptosis and differentiation in human leukaemia cells. Biochem J. 2012;443(3):735-746.

5. Shi Y, Jia B, Xu W, et al. Chidamide in relapsed or refractory peripheral $\mathrm{T}$ cell lymphoma: a multicenter real-world study in China. J Hematol Oncol. 2017;10(1):69.

6. He M, Qiao Z, Wang Y, et al. Chidamide inhibits aerobic metabolism to induce pan- creatic cancer cell growth arrest by promoting Mcl-1 degradation. PLoS One. 2016; 11(11):e0166896.

7. Hu X, Wang L, Lin L, et al. A phase I trial of an oral subtype-selective histone deacetylase inhibitor, chidamide, in combination with paclitaxel and carboplatin in patients with advanced non-small cell lung cancer. Chin J Cancer Res. 2016;28(4):444451.

8. Minami J, Suzuki R, Mazitschek R, et al. Histone deacetylase 3 as a novel therapeutic target in multiple myeloma. Leukemia. 2014;28(3):680-689.

9. Harada $\mathrm{T}$, Ohguchi $\mathrm{H}$, Grondin Y, et al. HDAC3 regulates DNMT1 expression in multiple myeloma: therapeutic implications. Leukemia. 2017:31(12):2670-2677.

10. Cantley MD, Fairlie DP, Bartold PM, Marino V, Gupta PK, Haynes DR. Inhibiting histone deacetylase 1 suppresses both inflammation and bone loss in arthritis. Rheumatology (Oxford). 2015; 54(9):1713-1723.

11. Dou C, Li N, Ding N, et al. HDAC2 regu- lates FoxO1 during RANKL-induced osteoclastogenesis. Am J Physiol Cell Physiol. 2016;310(10):C780-787

12. Garcia-Gomez A, Ocio EM, Crusoe E, et al. Dasatinib as a bone-modifying agent: anabolic and anti-resorptive effects. PLoS One. 2012;7(4):e34914

13. Tomimori Y, Mori $\mathrm{K}$, Koide $\mathrm{M}$, et al. Evaluation of pharmaceuticals with a novel 50-hour animal model of bone loss. J Bone Miner Res. 2009;24(7):1194-1205.

14. Paton-Hough J, Chantry AD, Lawson MA. A review of current murine models of multiple myeloma used to assess the efficacy of therapeutic agents on tumour growth and bone disease. Bone. 2015;77:57-68.

15. Hurchla MA, Garcia-Gomez A, Hornick MC, et al. The epoxyketone-based proteasome inhibitors carfilzomib and orally bioavailable oprozomib have anti-resorptive and bone-anabolic activity in addition to anti-myeloma effects. Leukemia. 2013; 27(2):430-440.

16. Lane NE, Yao W, Nakamura MC, et al. Mice 
Therapeutic effects of chidamide on myeloma-associated bone

lacking the integrin beta 5 subunit have accelerated osteoclast maturation and increased activity in the estrogen-deficient state. J Bone Miner Res. 2005;20(1):58-66.

17. Pain T, Garcia-Gomez A, GonzalezMendez L, et al. The Novel Pan-PIM Kinase Inhibitor, PIM447, Displays Dual Antimyeloma and Bone-Protective Effects, and Potently Synergizes with Current Standards of Care. Chin Cancer Res. 2017;23(1):225-238.

18. Tang J, Yah H, Zhuang S. Histone deacetylases as targets for treatment of multiple diseases. Chin Sci (Lond). 2013;124(11):651-662.

19. Laubach JP, San-Miguel JF, Hungria V, et al. Deacetylase inhibitors: an advance in myeloma therapy? Expert Rev Hematol. 2017;10(3):229-237.

20. Seta E, Yoshida M. Erasers of histone acetylation: the histone deacetylase enzymes. Cold Spring Herb Perspect Biol. 2014;6(4):a018713.

21. Pavithra L, Mukherjee S, Sreenath K, et al. SMAR1 forms a ternary complex with p53MDM2 and negatively regulates p53-medited transcription. J Mol Biol. 2009; 388(4):691-702.

22. Lesina M, Kurkowski MU, Lues K, et al. Stat3/Socs3 activation by IL- 6 transsignaling promotes progression of pancreatic intraepithelial neoplasia and development of pancreatic cancer. Cancer Cell. 2011;19(4):456469.

23. Given KS, Sikka S, Surana R, et al. Targeting the STAT3 signaling pathway in cancer: role of synthetic and natural inhibitors. Biochim Biophys Anta. 2014; 1845(2):136-154.

24. Kiang P, Chen SH, Ching CK, et al. Platelet factor 4 induces cell apoptosis by inhibition of STAT3 via up-regulation of SOCS3 expression in multiple myeloma. Haematological. 2013;98(2):288-295.

25. Ortega-Molina A, Boss IW, Canella A, et al. The histone lysine methyltransferase KMT2D sustains a gene expression program that represses B cell lymphoma developmont. Nat Med. 2015;21(10):1199-1208.

26. $\mathrm{Hu} Y$, Benedict MA, Wu D, Inohara $N$, Nunez G. Bcl-XL interacts with Apaf-1 and inhibits Apaf-1-dependent caspase-9 activatimon. Proc Natl Aced Sci USA. 1998;95(8):4386-4391.

27. Tan E, Weiss BM, Mona E, Horde N, Choyke PL, Landgren O. Current and future imaging modalities for multiple myeloma and its perecursor states. Leek Lymphoma. 2011;52(9): 1630-1640.

28. Dotterweich J, Schlegelmilch K, Keller A, et al. Contact of myeloma cells induces a characteristic transcriptome signature in skeletal precursor cells. Implications for myeloma bone disease. Bone. 2016;93:155-166.

29. Kawano Y, Moschetta M, Manier S, et al. Targeting the bone marrow microenvironmont in multiple myeloma. Immunol Rev. 2015;263(1):160-172.

30. Cantley MD, Zannettino ACW, Bartold PM, Fairlie DP, Haynes DR. Histone deacetylases (HDAC) in physiological and pathological bone remodelling. Bone. 2017; 95:162-174.

31. Phat L, Kaiser B, Roma A, et al. HDAC3 and HDAC7 have opposite effects on osteoclast differentiation. J Biol Chem. 2011;286(14):12056-12065.

32. Blixt NC, Faulkner BK, Astleford K, et al. Class II and IV HDACs function as inhibitors of osteoclast differentiation. PLoS One. 2017;12(9):e0185441.

33. Sun W, Zhao C, Li Y, et al. Osteoclastderived microRNA-containing exosomes selectively inhibit osteoblast activity. Cell Discov. 2016;2:16015.

34. Wallet S, Mukherjee S, Vaghela N, et al. Activin A promotes multiple myelomainduced osteolysis and is a promising target for myeloma bone disease. Proc Natl Arad Sci USA. 2010;107(11):5124-5129.

haematological | 2018; 103(8)

1379 